\title{
Carbonic anhydrase IX is a clinically significant tissue and serum biomarker associated with renal cell carcinoma
}

\author{
MARTINA TAKACOVA ${ }^{1,2}$, MARIA BARTOSOVA ${ }^{1}$, LUCIA SKVARKOVA ${ }^{1}$, MIRIAM ZATOVICOVA ${ }^{1}$, \\ IVANA VIDLICKOVA ${ }^{3}$, LUCIA CSADEROVA ${ }^{1,2}$, MONIKA BARATHOVA ${ }^{1}$, JAN BREZA Jr ${ }^{4}$, \\ PETER BUJDAK ${ }^{4}$, JAROMIR PASTOREK $^{1}$, JAN BREZA Sr $^{4}$ and SILVIA PASTOREKOVA ${ }^{1}$ \\ ${ }^{1}$ Department of Molecular Medicine, Institute of Virology and ${ }^{2}$ Center for Molecular Medicine, Slovak Academy of Sciences; \\ ${ }^{3}$ Department of Molecular Biology, Faculty of Natural Sciences, Comenius University; \\ ${ }^{4}$ Department of Urology, Derer's University Hospital, Bratislava, Slovakia
}

Received February 3, 2012; Accepted June 15, 2012

DOI: $10.3892 / \mathrm{ol} .2012 .1001$

\begin{abstract}
Carbonic anhydrase IX (CA IX) is regarded as one of the most prominent markers of tumor hypoxia with potential to serve as a diagnostic biomarker, prognostic indicator as well as tumor therapeutic target. The aim of the present study was to perform an in-depth analysis of CA IX expression in blood and tissue samples and to evaluate the significance of CA IX status for different renal cell carcinomas (RCCs). The expression of CA IX was determined in blood and tissue samples from 74 kidney cancer patients using reverse transcription polymerase chain reaction (RT-PCR), enzyme-linked immunosorbent assay (ELISA), Western blotting (WB) and immunohistochemistry (IHC). The CA IX status was correlated with RCC type and tumor stage. IHC and WB provided evidence for a significantly higher expression of CA IX in clear cell RCC (CCRCC) specimens compared to other RCCs. RT-PCR assay revealed that $32.42 \%$ of all RCC patients possess $C A 9$-positive cells in peripheral blood and three-quarters of CA9-positive patients were diagnosed with CCRCC. When the patients were subdivided according to tumor stage, decreased positivity was observed with higher tumor stage (50\% in T1 vs. $17 \%$ in T3). Serum CA IX levels determined
\end{abstract}

Correspondence to: Professor Silvia Pastorekova, Department of Molecular Medicine, Institute of Virology, Slovak Academy of Sciences, Dubravska cesta 9, Bratislava 845 05, Slovakia

E-mail: virusipa@savba.sk

Abbreviations: CA IX, carbonic anhydrase IX protein; CA9, carbonic anhydrase IX gene; HIF, hypoxia-inducible factor; RCC, renal cell carcinoma; CCRCC, clear cell RCC; PRCC, papillary RCC; CHRCC, chromophobe RCC; BT, benign tumor; RT-PCR, reverse transcription polymerase chain reaction; ELISA, enzyme-linked immunosorbent assay; WB, Western blotting; IHC, immunohistochemistry

Key words: carbonic anhydrase IX, renal cell carcinoma, biomarker, circulating tumor cells by ELISA were significantly higher in CCRCC patients than in non-CCRCC. A significant association between s-CA IX and CCRCC tumor stage was also determined (T1-87.51 vs. T3-341.98 pg/ml, p=0.046). We demonstrated that the CA IX expression profiles in blood and tissue samples from 74 kidney cancer patients are closely correlated with their histological subtypes. This is the first study reporting CA IX expression in blood and tissue samples from kidney cancer patients determined by four different methods.

\section{Introduction}

Renal cell carcinoma (RCC), the most lethal type of the urological cancers, accounts for $3 \%$ of all adult malignancies (1). There are four main histological subtypes of RCC: clear cell (CCRCC), papillary (PRCC; divided into two groups: type I and type II) and chromophobe (CHRCC) carcinoma. The great majority of RCC cases are clear cell carcinoma, which is characterized by the bi-allelic inactivation of the von Hippel-Lindau (VHL) gene (2). This genetic event leads to the stabilization of the $\alpha$-subunit of hypoxia-inducible factor 1 (HIF-1). HIF-1 is a key transcriptional regulator that promotes cell growth and survival under hypoxic conditions. Its activation subsequently leads to the overexpression of multiple genes responsible for tumor angiogenesis, cell migration, glucose transport, glycolysis and $\mathrm{pH}$ control. Among the genes, carbonic anhydrase IX (CA9) is one of the most prominent markers of tumor hypoxia mainly due to the unique localization of its hypoxia response element at the -10/-3 position from the transcription start site of the CA9 gene (3).

CA IX protein, as a member of the carbonic anhydrase family, catalyzes the reversible conversion of carbon dioxide to bicarbonate and protons and is thus involved in ion transport and $\mathrm{pH}$ control (4). CA IX is a transmembrane protein which is ectopically expressed in various types of human cancer (e.g., cervical, lung, breast, and head and neck). Moreover, tumors with a high CA IX expression exhibit a more aggressive phenotype and have a poor prognosis. Paradoxically, CA IX overexpression in CCRCC appears to be an early event and is associated with better prognosis (5). The clinical and 
prognostic significance of CA IX in RCC have been extensively evaluated. CA IX is regarded as one of the most promising biomarkers in clear cell RCC as it has a conclusive diagnostic, prognostic, as well as therapeutic potential. In this study, we utilized all available clinical material from 74 kidney cancer patients with a focus on determining the status of CA IX. For this purpose, we investigated the expression of CA IX in tumor tissue samples using Western blotting (WB), ELISA and immunohistochemistry. Moreover, we performed two different blood-based assays: RT-PCR for $C A 9$ gene expression in circulating tumor cells (CTCs) and ELISA for CA IX protein quantification in serum samples.

\section{Materials and methods}

Study subjects. Between March 2009 and May 2011, 74 patients with renal tumors were treated at the Department of Urology, Derer's University Hospital Bratislava, Slovakia. Seventy patients underwent partial or radical nephrectomy for malignant renal tumors and four patients for benign renal tumors (three were oncocytomas and one was angiomyolipoma). Of the 70 RCC patients, $57(81.4 \%)$ had conventional, 10 (14.3\%) papillary, and $3(4.3 \%)$ chromophobe histological features. Patient ages ranged from 29 to 80 years, with a mean of 59.83 years. Patients included 47 males and 27 females. Participants of this study were informed, and oral consent was obtained from each patient.

Collection of samples. Blood samples were collected prior to surgery. Immediately after collection, $200 \mu \mathrm{l}$ of peripheral blood were rapidly agitated with $800 \mu 1$ of InstaPure reagent (Eurogentec, Seraing, Belgium) and processed for RNA extraction with $175 \mu \mathrm{l}$ chloroform. Following centrifugation, the aqueous phase was transferred to a fresh tube and an equal volume of isopropanol was added and mixed. Precipitated pellets of RNA were washed firstly with $70 \%$ ethanol and then absolute ethanol. At the end of the procedure, RNA pellets were dried and dissolved in $10 \mu \mathrm{l}$ of DEPC-treated water. For serum isolation, the blood tube was centrifuged at 2500 $\mathrm{x} \mathrm{g}$ for $15 \mathrm{~min}$ at $4^{\circ} \mathrm{C}$ and the serum samples were stored at $-20^{\circ} \mathrm{C}$ prior to use. Tissue samples were obtained from surgical nephrectomy specimens and processed within $1 \mathrm{~h}$ following surgical resection. Portions of the fresh tissues from RCCs and normal renal tissues were frozen (in liquid nitrogen) and stored at $-80^{\circ} \mathrm{C}$.

$R T$-PCR. Reverse transcription of isolated RNA dissolved in $10 \mu \mathrm{l}$ DEPC-treated water was performed using a High Capacity cDNA Reverse Transcription Kit (AB Applied Biosystems, Foster City, CA, USA). RT-PCR was carried out with $0.5 \mu \mathrm{l}$ from a total of $20 \mu \mathrm{l}$ of cDNA with $C A 9$-specific primers and primers for $\beta$-actin that served as an internal standard. The primers were as follows ( $\mathrm{S}$, sense; $\mathrm{A}$, antisense): CA9S 5'-TATCTGCACTCCTGCCCTCTG-3' and CA9A 5'-CACAGGGTGTCAGAGAGGGTGT-3' (154 bp product); $\beta$-actinS 5'-CCAACCGCGAGAAGATGACC-3' and $\beta$-actinA 5'-GATCTTCATGAGGTAGTCAGT-3' (236 bp product). RT-PCR was carried out with DreamTaq ${ }^{\mathrm{TM}}$ Green PCR Master mix (Fermentas, St. Leon-Rot, Germany). Following the initial denaturation at $94^{\circ} \mathrm{C}$ for $3 \mathrm{~min}$, the amplification program was set as follows: denaturation at $94^{\circ} \mathrm{C}$ for $30 \mathrm{sec}$, annealing at $60 / 65^{\circ} \mathrm{C}\left(\beta\right.$-actin/CA9) for $30 \mathrm{sec}$, and extension at $72^{\circ} \mathrm{C}$ during $30 \mathrm{sec}$ for a total of 30/35 cycles ( $\beta$-actin/CA9), and final extension at $72^{\circ} \mathrm{C}$ for $7 \mathrm{~min}$. Positive RT-PCR control was provided by cDNA from HeLa cells. The PCR reactions were repeated three times.

Western blotting. For Western blotting analysis, frozen tissue specimens were homogenized in ice-cold lysis buffer $(1 \%$ Triton X-100; $150 \mathrm{mM} \mathrm{NaCl} ; 50 \mathrm{mM}$ Tris, $\mathrm{pH} \mathrm{7.5;0.5 \%}$ Nonidet P-40; $50 \mathrm{mM} \mathrm{NaF}$ ) containing inhibitors of proteases (Roche Applied Science, Mannheim, Germany). The protein concentration was determined using the BCA protein assay reagent (Pierce, Rockford, IL, USA). Total protein extracts $(100 \mu \mathrm{g} /$ lane) were separated in 10\% SDS-PAGE and transferred onto PVDF membranes (Immobilon ${ }^{\mathrm{TM}}$-P, Millipore, Billerica, MA, USA). For CA IX detection, the membrane was incubated with M75 primary antibody (6) and diluted 1:2 in blocking buffer for $1 \mathrm{~h}$. Secondary anti-mouse peroxidase-conjugated antibody (Dako, Glostrup, Denmark) was diluted 1:5000 in blocking buffer. As a loading control, the membrane was probed with anti-actin antibody (Santa Cruz Biotechnology Inc., Santa Cruz, CA, USA) and the polyclonal rabbit anti-goat IgG-HRP (Dako). Following treatment, the membrane was washed and developed by enhanced chemiluminescence using an ECL kit (Amersham Pharmacia Biotech, Buckinghamshire, UK).

ELISA. Serum samples were initially analyzed with commercially available ELISA DuoSet ${ }^{\circledR}$ Human Carbonic Anhydrase IX (R\&D Systems, Inc., Minneapolis, MN, USA) according to the manufacturer's instructions. Quantification was performed with V/10 antibody (7) which replaced capture antibody in the ELISA kit supplied by R\&D. Samples were assayed in duplicate and repeated three times. For CA IX tissue quantification, sandwich ELISA with V/10 as a capture antibody was performed. The same protein lysates as in Western blotting were used and the final CA IX concentration was calculated from $100 \mu \mathrm{g}$ of total proteins.

Immunohistochemistry. Dissected tissues were embedded in paraffin according to the standard histological procedure. Immunohistochemistry was performed on an automated immunostainer (Dako Autostainer) using DakoCytomation EnVision ${ }^{\oplus}+$ System-HRP (DAB) according to the manufacturer's instructions. Sections $(4 \mu \mathrm{m})$ were incubated with M75 antibody (hybridoma medium diluted 1:100) for $1 \mathrm{~h}$ at room temperature. Staining was visualized with DAB solution for 1 min with 3,3'-diaminobenzidine as a chromogenic substrate. Finally, the sections were counterstained with Mayer's hematoxylin and mounted in Aquamount (Merck, Darmstadt, Germany). The stained sections were examined using a Leica DM4500B microscope and images were captured by a Leica DFC480 camera. CA IX is a membrane-associated antigen and thus, cells exhibiting clear and sharp membrane staining were interpreted as CA IX-positive. The percentage of CA IX-stained pixels was evaluated on the entire tissue section with ImageJ 1.38x software (Rasband, W.S., ImageJ, U.S. National Institutes of Health, Bethesda, MD, USA, http://rsb.info.nih. gov/ij/, 1997-2007). ImageJ is able to create density histograms 
Table I. Patient and tumor characteristics.

\begin{tabular}{lc}
\hline Characteristics & No. \\
\hline Patient (No.) & 74 \\
Male & 47 \\
Female & 27 \\
Mean age (years) & 59.83 \\
Male & 58.92 \\
Female & 60.74 \\
Histological subtype (No.) & \\
Clear cell RCC & 57 \\
Papillary RCC & 10 \\
Chromophobe RCC & 3 \\
Benign tumor & 4 \\
T classification (in CCRCC) & \\
T1 & 28 \\
T2 & 5 \\
T3 & 24 \\
T4 & 0 \\
\hline
\end{tabular}

and calculate area and pixel value statistics of user defined selections. The proportion as well as intensity of CA IX-stained pixels was calculated as an average from 10 images captured from each tissue sample. Specimens that had no evidence of specific immunostaining were marked as negative.

Statistical analysis. Results were analyzed by the two-tailed unpaired t test (Student's t-test). $\mathrm{P}<0.05$ was considered statistically significant. The correlation between different variables was examined using the Pearson's correlation coefficient.

\section{Results}

Study subjects. A total of 74 patients were included in this study (Table I). CA IX expression was evaluated in blood samples using RT-PCR and ELISA as well as in tissue specimens using WB and immunohistochemistry.

CA IX as a biomarker in blood-based assays. RT-PCR for CA9 detection was performed on blood samples obtained from 74 patients. Cells expressing CA9 were detected in 24 of $74(32.43 \%)$ kidney cancer patients. The CA9-positive samples were obtained from patients with malignant renal tumors: 18 (75\%) were diagnosed with CCRCC, 4 (16.67\%) with PRCC and $2(8.33 \%)$ with CHRCC. Four patients with benign tumors (BTs) were $C A 9$-negative. No blood samples from the healthy controls were found to be positive for $C A 9$ (data not shown) and thus, the specificity was $100 \%$ in our study.

From 57 CCRCC patients, 18 (32\%) were found to be positive for $C A 9$ expression in the peripheral blood. Decreased positivity was observed with the higher tumor stage (Table II). Patients with stage T3 exhibited CA9-positive CTCs in $17 \%$ of the cases compared with $50 \%$ for the patients with $\mathrm{T} 1$ disease. The small number of specimens in stage T2 limited any statistical evaluation.
Table II. RT-PCR results of CA9 expression in circulating tumor cells according to the histological subtype of RCC and tumor stage of CCRCC patients.

\begin{tabular}{lcc}
\hline Subtype & CA9-positive & CA9-negative \\
\hline CCRCC & 18 & 39 \\
T1 & 14 & 14 \\
T2 & 0 & 5 \\
T3 & 4 & 20 \\
T4 & 0 & 0 \\
PRCC & 4 & 6 \\
CHRCC & 2 & 1 \\
BT & 0 & 4 \\
\hline
\end{tabular}

RCC, renal cell carcinoma; CCRCC, clear cell renal cell carcinoma; PRCC, papillary renal cell carcinoma; CHRCC, chromophobe renal cell carcinoma; BT, benign tumor.

Soluble forms of CA IX (s-CA IX) shed from renal tumors were initially determined by commercially available ELISA (DuoSet ${ }^{\circledR}$ Human Carbonic Anhydrase IX, R\&D Systems). Although a significant association between serum CA IX and the histological subtype of RCC was observed, the association with tumor stage did not reach statistical significance (Table III). Therefore, the following evaluation was performed with V/10 as a capture antibody. The s-CA IX levels in serum samples from CCRCC patients were found to be significantly higher compared to samples in the non-CCRCC $(\mathrm{p}=0.002)$ and benign tumors $(\mathrm{p}=0.002)$ (Table III). The mean level of $\mathrm{s}-\mathrm{CA}$ IX was $209.22 \mathrm{pg} / \mathrm{ml}$ in 57 CCRCC patients, $28.78 \mathrm{pg} / \mathrm{ml}$ in 13 non-CCRCC (PRCC and CHRCC) patients and $21.84 \mathrm{pg} /$ $\mathrm{ml}$ in 4 BTs. Stratification of the CCRCC patients according to the tumor stage revealed a significant association between $\mathrm{s}-\mathrm{CA}$ IX and tumor stage $(\mathrm{p}=0.046)$ with the mean value for stage T1 and T3 $87.51 \mathrm{pg} / \mathrm{ml}$ and $341.98 \mathrm{pg} / \mathrm{ml}$, respectively.

CA IX as a tissue marker. Seventy-four frozen tissue specimens were used to determine the expression of CA IX by Western blotting. The same twin band of CA IX (54 and $58 \mathrm{kDa}$ ) as in protein lysates from tumor cell cultures was observed in the protein lysates from tissues. CA IX expression was assessed in $74.32 \%$ of tumor tissue samples. The majority of the CA IX-positive samples (50 of 55) were CCRCC tumors. The rest of the positive tissues were four papillary RCCs and one chromophobe RCC. Specimens from BTs were negative. Within the CCRCC group, $88 \%$ positivity for CA IX expression was obtained. By contrast, parallel tissue samples from healthy kidneys demonstrated a complete absence of CA IX protein bands (Fig. 1).

For CA IX quantification, the same protein lysates from 74 tumor tissues were analyzed by ELISA. According to the protein standard, the final concentration of CA IX in $100 \mu \mathrm{g}$ of total proteins was calculated. As expected, CA IX tissue levels were significantly higher in CCRCC than in non-CCRCC and BTs (6057.83 pg vs. 172.82 and 6057.83 pg vs. 20.79 pg/ml, $\mathrm{p}<0.001$ and $\mathrm{p}<0.001)$. The CA IX expression was significantly 
Table III. Relationship between CA IX levels (measured by ELISA in serum and tissue) and clinicopathological variables.

\begin{tabular}{|c|c|c|c|}
\hline & $\begin{array}{l}\text { Serum CA IX }(\mathrm{pg} / \mathrm{ml}) \\
\text { V/10 }\end{array}$ & $\begin{array}{l}\text { Serum CA IX }(\mathrm{pg} / \mathrm{ml}) \\
\text { R\&D }\end{array}$ & $\begin{array}{c}\text { Tissue CA IX (pg/100 } \mu \mathrm{g} \\
\text { of total proteins) }\end{array}$ \\
\hline CCRCC (all stages) & 209.22 & 308.53 & 6057.83 \\
\hline CCRCC stage T1 & 87.51 & 269.67 & 8278.05 \\
\hline CCRCC stage T3 & 341.98 & 312.39 & 4478.82 \\
\hline Non-CCRCC & 28.78 & 73.49 & 172.82 \\
\hline BT & 21.84 & 18.94 & 20.79 \\
\hline $\mathrm{p}$-value & $\begin{array}{c}\text { CCRCC vs. non- } \\
\text { CCRCC, } 0.002 \\
\text { CCRCC vs. BT, } 0.002 \\
\text { T1 vs. T3 CCRCC, } \\
0.046\end{array}$ & $\begin{array}{c}\text { CCRCC vs. non- } \\
\text { CCRCC, } 0.01 \\
\text { CCRCC vs. BT, } 0.002 \\
\text { T1 vs. T3 CCRCC, } \\
0.830\end{array}$ & $\begin{array}{c}\text { CCRCC vs. non-CCRCC, } \\
<0.001 \\
\text { CCRCC vs. BT, }<0.001 \\
\text { T1 vs. T3 CCRCC, } 0.043\end{array}$ \\
\hline
\end{tabular}

RCC, renal cell carcinoma; CCRCC, clear cell renal cell carcinoma; PRCC, papillary renal cell carcinoma; CHRCC, chromophobe renal cell carcinoma; BT, benign tumor.

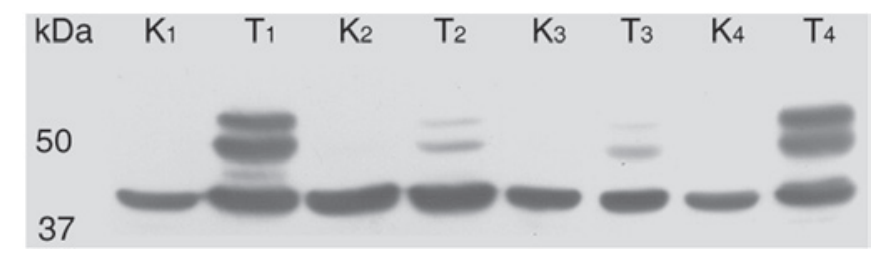

Figure 1. CA IX expression in RCC tissues analyzed by Western blotting. Expression of CA IX in the tumor tissues from various RCCs was analyzed by Western blotting using the specific monoclonal antibody M75. Tumor samples (T) containing a twin band (54 and $58 \mathrm{kDa}$ ) which represents the CA IX protein were marked as positive. Normal kidney tissue $(\mathrm{K})$ showed a complete absence of CA IX. Anti-actin antibody was used as a loading control $(42 \mathrm{kDa})$.

higher in stage $\mathrm{T} 1$ when compared with stage $\mathrm{T} 3(\mathrm{p}=0.043)$ (Table III). Additionally, there was a trend toward a higher detection of CA9 mRNA in CTCs using RT-PCR in a group of T1 tumors (the mean value is $8278.05 \mathrm{pg}$ ) exhibiting a significantly higher mean CA IX expression when compared with T3 tumors (4478.82 pg) (Fig. 2). Despite a relative increased positivity in $\mathrm{T} 1$ tumors $>4 \mathrm{~cm}$ and decreased positivity in T3 tumors $>7 \mathrm{~cm}$, the p-value did not reach a statistical cut-off (data not shown).

CA IX expression in tumor tissues was also evaluated by immunohistochemistry (Fig. 3). Overall, $82.43 \%$ of 74 tumor specimens exhibited CA IX expression. CA IX expression was detected in $93 \%$ (53 of 57) of CCRCC and in $60 \%$ (6 of 10) of papillary RCC. One positive tumor was observed from chromophobe RCCs and benign tumors but the overall small number of specimens in these groups limits any evaluation. The proportion of CA IX-stained pixels from the entire image as well as staining intensity of CA IX-positive pixels were analyzed as an average from 10 images captured from each tissue section. Proportions of CA IX-stained pixels were $6.30 \%$ in CCRCC, $0.24 \%$ in non-CCRCC and $0.25 \%$ in BT (Table IV). The CA IX status determined by IHC was significantly higher in CCRCC than in non-CCRCC $(\mathrm{p}<0.001)$ and

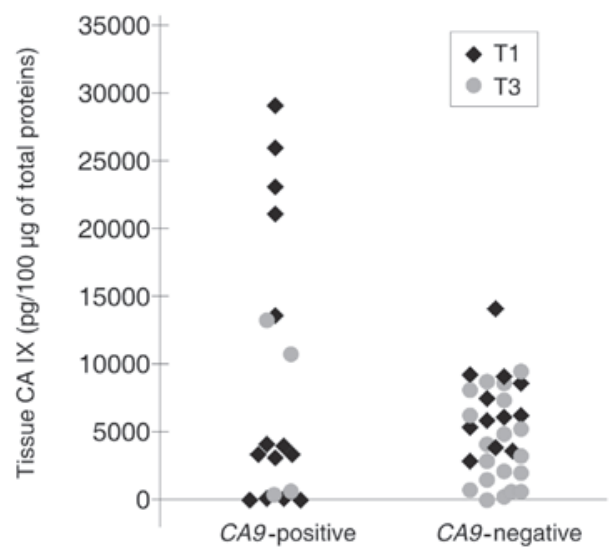

Figure 2. Correlation between circulating tumor cell detection and CA IX expression in tumor tissue. CCRCC patients were stratified according to tumor stage and detection of CA9 mRNA in peripheral blood samples using RT-PCR (x-axis) was compared with tissue CA IX quantification (in $\mathrm{pg} / 100 \mu \mathrm{g}$ of total proteins) using ELISA (y-axis).

BT ( $<<0.001)$, respectively. The mean value of CA IX staining intensity was 119.91 in CCRCC and was significantly higher than in non-CCRCC $(72.58, \mathrm{p}=0.035)$ and in BT $(29.15$, $\mathrm{p}=0.05)$. Within the CCRCC group, there was a non-significant trend towards a decreased proportion of CA IX staining as well as CA IX intensity in stage T3 when compared with T1. CA IX staining in RCC samples was positively correlated with CA IX tissue level measured by ELISA (Pearson's correlation coefficient $=0.66$ ).

\section{Discussion}

Despite the accumulating data obtained on the biological and clinical features of kidney cancer, management of tumor patients remains problematic. Use of more effective targeted therapy compared to conventional therapy is crucial. However, further progress can only be achieved through the identification and validation of relevant biomarkers for the rational 
Table IV. CA IX expression determined by immunohistochemistry and patient clinical characteristics.

\begin{tabular}{|c|c|c|c|c|}
\hline & $\begin{array}{l}\text { CA IX- } \\
\text { positive }\end{array}$ & $\begin{array}{l}\text { CA IX- } \\
\text { negative }\end{array}$ & $\begin{array}{l}\text { Proportion of CA IX- } \\
\text { stained pixels }(\%)\end{array}$ & $\begin{array}{l}\text { Intensity of CA IX- } \\
\text { stained pixels }\end{array}$ \\
\hline CCRCC (all stages) & 53 & 4 & 6.30 & 119.91 \\
\hline CCRCC stage T1 & 26 & 2 & 6.68 & 124.03 \\
\hline CCRCC stage T3 & 22 & 2 & 5.33 & 115.20 \\
\hline Non-CCRCC & 7 & 6 & 0.24 & 72.58 \\
\hline BT & 1 & 3 & 0.25 & 29.15 \\
\hline $\mathrm{p}$-value & & & $\begin{array}{c}\text { CCRCC vs. non- } \\
\text { CCRCC },<0.001 \\
\text { CCRCC vs. BT, }<0.001 \\
\text { T1 vs. T3 CCRCC, } \\
0.396\end{array}$ & $\begin{array}{c}\text { CCRCC vs. non- } \\
\text { CCRCC, } 0.035 \\
\text { CCRCC vs. BT, } 0.050 \\
\text { T1 vs. T3 CCRCC, } \\
0.422\end{array}$ \\
\hline
\end{tabular}

RCC, renal cell carcinoma; CCRCC, clear cell renal cell carcinoma; PRCC, papillary renal cell carcinoma; CHRCC, chromophobe renal cell carcinoma; BT, benign tumor.
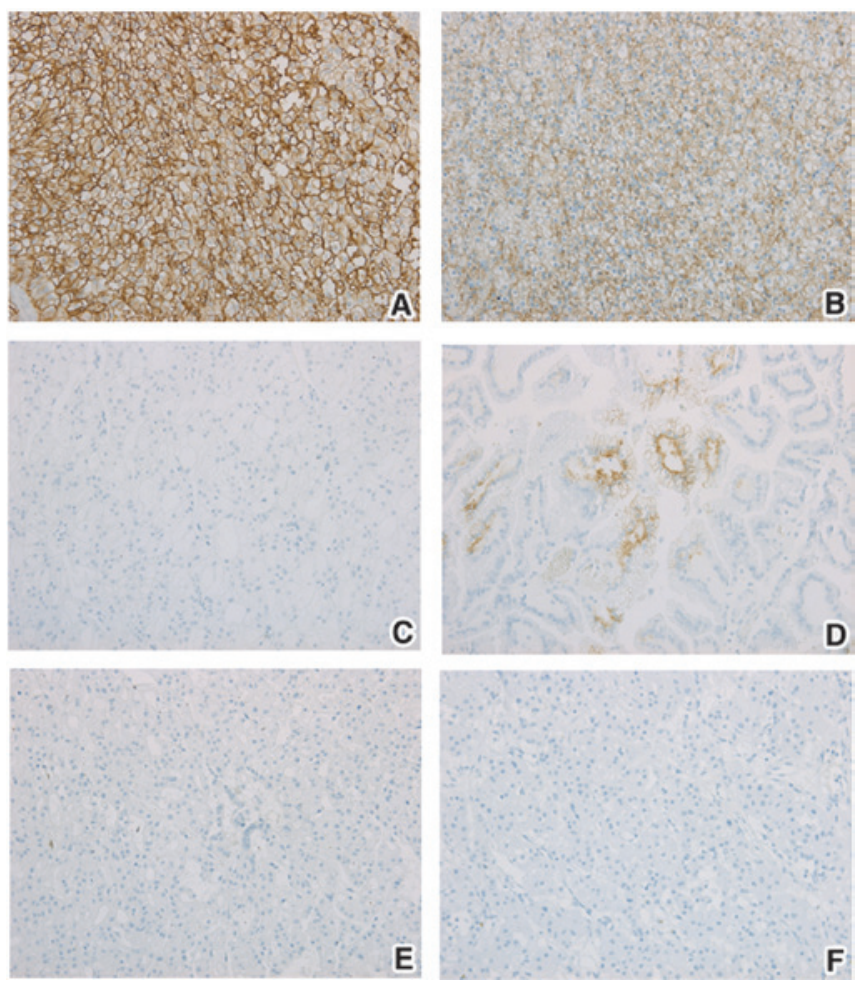

Figure 3. Immunohistochemical staining for CA IX expression in RCC tumor samples. Representative view of CA IX staining from (A,B and C) clear cell, (D) papillary and (E) chromophobe RCC as well as (F) oncocytoma.

stratification of patients and selection of proper treatment strategy. Although CA IX is one of the most studied surface antigens in RCC, attention has focused on the evaluation of CA IX for CCRCC patients. Several studies demonstrated that a high CA IX expression (unlike in non-renal tumors) correlates with better clinical outcomes and is also able to predict response to high-dose IL-2 therapy in advanced stage CCRCC $(5,8)$. In light of these facts, we performed a detection and quantification of CA IX in blood as well as tissue specimens from 74 kidney cancer patients to clarify its clinical importance.

The first method we used was RT-PCR detection of circulating tumor cells (CTCs) in the peripheral blood. The spread of neoplastic cells from primary tumor to distant sites is a crucial step in the metastasis process and, thus, the detection of CTCs may provide evidence about disease aggression. Bluemke and colleagues screened the status of CTCs in the blood samples from renal carcinoma patients and revealed that the presence of CTCs correlated with the lymph node status and synchronous metastasis (9). The ability to detect CTCs by analyzing the expression of CA9 as a tumor-specific biomarker in blood samples has previously been reported (10-13). RT-PCR for CA9 is a highly specific and sensitive technique for detecting CTCs shed from renal carcinomas. In this study, almost $33 \%$ of the 74 patients tested by RT-PCR had CA9-positive CTCs. The majority of the patients were diagnosed with conventional RCC. Additionally, with higher tumor stage, there was a trend towards reduced or even absent CA9 mRNA in blood specimens. Several studies, however, were not able to correlate results obtained from RT-PCR to the clinical characteristics of the patients. A possible explanation for this lies in the specificity and sensitivity of this type of assay, which is heavily dependent on the set of primers that are used.

Thus, the first prerequisite for acquisition of correct results is in primer design which reflects the exon-intron structure of the CA9 gene (14). Furthermore, Barathova et al demonstrated that the $C A 9$ gene expression involves alternative splicing and thus, the alternatively spliced (AS) variant of CA9 mRNA lacking exons 8-9 exists. In contrast to fulllength (FL) CA9 mRNA, the AS variant is detected in normal tissues and independently from the levels of hypoxia (15). Therefore, it may produce false-positive results and affect the clinical value of CA IX as a marker of hypoxia $(16,17)$. Additional steps, including preference of small volume of blood samples $(200 \mu \mathrm{l})$ prior to enrichment from larger blood volumes and rapid RNA isolation following homogenization 
of blood samples with InstaPure reagent, are crucial steps for obtaining significant results in this study. McKiernan et al assessed CA9-expressing cells in $49 \%$ of a total of $37 \mathrm{RCC}$ patients. Using the primers designated against exons 1 and 5 , they detected the two variants of $C A 9$ mRNA and, therefore, they did not reveal any correlation between $C A 9$ expression and age, gender or tumor grade (10). The same set of primers with contrasting results was used in a further two studies. The first one revealed only $19 \%$ positivity for $C A 9$ in a group of 59 patients with RCC (11). However, almost $32 \%$ positivity for CA9 in peripheral blood samples was achieved by Gilbert et al (12). Considering that these authors used similar methodology and comparable patient groups, there is no explanation for the discrepancies. However, the second study provides evidence that the estimated 5-year disease-free survival for patients with detectable CA9 mRNA in the peripheral blood was $39.5 \%$ in comparison with $88.1 \%$ for $C A 9$-negative patients.

Several studies have examined the potential clinical utility of s-CA IX in the serum from RCC patients for prognostic and predictive applications (18-23). s-CA IX is a shorter form of CA IX $(50 / 54 \mathrm{kDa})$ which is released into the culture medium or into the body fluids via a metalloprotease-dependent process regulated by TACE/ADAM17 (24). Zavada et al first described the detection of s-CA IX in the serum and urine of patients with RCC (18). In this study, two different capture antibodies were employed to compare the results. Initially, we used the commercially available CA IX ELISA kit from R\&D. s-CA IX in serum samples was then quantified using V/10 as a capture antibody (7). In both cases, we observed a significant correlation between serum CA IX and the histological subtype of RCC. Although lower mean values of s-CA IX were obtained, introduction of the V/10 antibody enabled us to reveal a significant association between the s-CA IX level and tumor stage in CCRCC patients. Our results are in accordance with the study of Li et al, who first reported a significant association between serum CA IX and stage, tumor grade and size in patients with CCRCC. In their study of 91 patients, the mean value of serum CA IX in patients with metastatic CCRCC (216.68 $\pm 67.02 \mathrm{pg} /$ ml) was significantly higher than that in localized CCRCC (91.65 $\pm 13.29 \mathrm{pg} / \mathrm{ml})$ (19). Similar CA IX values for CCRCC patients $(126.1 \mathrm{pg} / \mathrm{ml})$ with the same commercial ELISA kit for CA IX were reported by Zhou et al (20). However, the serum CA IX levels measured by ELISA did not correlate with the tissue levels of CA IX determined by ELISA or immunohistochemistry. By contrast, a significant trend for an association between serum CA IX and tumor expression assessed with immunohistochemistry was described by Papworth et al (21). Their results showed higher serum CA IX in CCRCC compared to other RCC types and a positive correlation of CA IX with tumor stage was revealed. However, these authors did not find a significant prognostic association between serum CA IX and the recurrence-free survival of CCRCC patients.

In addition to the blood-based assays, WB and immunohistochemistry may be used to determine the status of CA IX in tissue samples. Previously, several studies have demonstrated the expression of CA IX in kidney tumors by WB $(18,25)$. Our study has shown that the majority of CCRCC patients expressed CA IX protein in tumor tissue. By contrast, tissue specimens from normal kidney and BTs were completely CA IX-negative. Despite the fact that WB reliably distinguishes CCRCC (as strongly CA IX-positive samples) from non-RCC tissues, it is clear that this method is not really suitable as a diagnostic test for a large amount of tissue samples. Furthermore, it is a time-consuming assay which is at best only semi-quantitative. Consequently, we performed ELISA to determine the level of CA IX expression in tumor tissues. Zhou et al have recently demonstrated that ELISA provides an objective and quantitative method for measurement of CA IX in renal tissues (20). Moreover, the tissue CA IX levels determined by ELISA highly correlated with the CA IX detected by immunohistochemistry. In agreement with Zhou et al, we found that CCRCC tissues demonstrated significantly higher CA IX levels than nonCCRCC and benign tumors. By contrast, we demonstrated a significantly higher CA IX expression for CCRCC in stage T1 (8278.05 pg/100 $\mu \mathrm{g}$ of total proteins) when compared with stage T3 tumors (4478.82 pg). Findings of the present study showed a trend to a higher CA IX expression in tumors larger than $4 \mathrm{~cm}$ in stage T1 and decreased positivity for T3 tumors larger that $7 \mathrm{~cm}$. However, no statistically significant difference was observed between them. These results are crucial particularly from the point of view of clinical application of ELISA in tissue. CA IX levels determined from smaller quantity of tumor tissue (when compared with IHC) may therefore serve as a method for stratification of RCC patients.

Immunohistochemistry, in contrast to $\mathrm{WB}$, has become the gold standard for measuring the CA IX expression in kidney tumors and thus, several studies have reported a prognostic significance of CA IX in $\operatorname{RCC}(5,8,26,27)$. However, the evaluation of CA IX in RCC tumors may be directly affected by the primary antibody or by the size of the tumor sample. Inasmuch as a small piece of tissue (for example as used in tissue microarrays) is not representative, we preferred an analysis of the whole tissue sections of RCC tumors. In this study, we confirmed that CA IX expression in tissue sections was correlated with the histological subtype of RCC. Therefore, we observed that $93 \%$ of CCRCC patients demonstrated CA IX expression. Additionally, we found that as the stage of CCRCC tumors increases, the staining for CA IX decreases. Our results are in accordance with Bui and colleagues, who first reported that diffuse membranous CA IX staining may be found in more than $90 \%$ of CCRCC patients (5). Diffuse intratumoral distribution is a result of VHL inactivation and thus, constitutive activation of HIF-1 (28). By contrast, in non-renal solid malignancies, the hypoxic activation of $\mathrm{HIF}-1 \alpha$ results in focal CA IX expression predominantly in the perinecrotic regions (3). In our study, we confirmed that CA IX is typically more highly expressed in clear cell RCCs than in non-CCRCC tumors and BTs. Moreover, the staining pattern of CA IX in papillary and chromophobe RCCs was not homogenous as in the case of CCRCC but rather fitted more to the hypoxia-induced pathway. Additionally, the staining intensity for CA IX was significantly higher in CCRCC than in non-CCRCC groups.

In conclusion, we have analyzed tissue as well as blood samples from a group of 74 kidney patients. Blood-based assays were performed for the detection of CA9 mRNA using RT-PCR or for s-CA IX protein shed from the existing renal tumor. In the current study, we describe the first use of specific primers for the full-length form of CA9 to detect the presence of CTCs in the peripheral blood of kidney cancer patients. The main finding of our study is that RT-PCR defined 
CA9 expression in CTCs as able to stratify patients with clear cell RCC into groups according to the tumor stage, with decreased or absent $C A 9$ expression being associated with worse prognosis. Therefore, RT-PCR detection of CA9 expression in the peripheral blood may indicate future prospects for the non-invasive diagnosis of RCC. Together with the quantification of CA IX in serum and tissue samples via ELISA, it may serve as a basis for optimal management of tumor patients and may be important for monitoring the progression and recurrence of the disease. Additional studies with larger cohorts of patients, along with relevant follow-up are required to confirm the clinical relevance and utility of the CA IX as a biomarker.

\section{Acknowledgements}

This study was supported by the grants from the Slovak Scientific Grant Agency (VEGA 2/0216/09, VEGA 2/0184/09 and VEGA 2/0152/12), from the 7th Framework program of the EU (Collaborative project METOXIA), and from the Research \& Development Operational Program funded by the ERDF (project ITMS 26240220062).

\section{References}

1. Jemal A, Siegel R, Xu J and Ward E: Cancer statistics, 2010. CA Cancer J Clin 60: 277-300, 2010

2. Gnarra JR, Tory K, Weng Y, et al: Mutations of the VHL tumour suppressor gene in renal carcinoma. Nat Genet 7 : 85-90, 1994.

3. Wykoff CC, Beasley NJ, Watson PH, et al: Hypoxia-inducible expression of tumor-associated carbonic anhydrases. Cancer Res 60: 7075-7083, 2000.

4. Svastova E, Hulikova A, Rafajova M, et al: Hypoxia activates the capacity of tumor-associated carbonic anhydrase IX to acidify extracellular pH. FEBS Lett 577: 439-445, 2004.

5. Bui MH, Seligson D, Han KR, et al: Carbonic anhydrase IX is an independent predictor of survival in advanced renal clear cell carcinoma: implications for prognosis and therapy. Clin Cancer Res 9: 802-811, 2003.

6. Pastorekova S, Zavadova Z, Kostal M, Babusikova O and Zavada J: A novel quasi-viral agent, MaTu, is a two-component system. Virology 187: 620-626, 1992.

7. Zat'ovicova M, Tarabkova K, Svastova E, et al: Monoclonal antibodies generated in carbonic anhydrase IX-deficient mice recognize different domains of tumour-associated hypoxiainduced carbonic anhydrase IX. J Immunol Methods 282: 117-134, 2003.

8. Atkins M, Regan M, McDermott D, et al: Carbonic anhydrase IX expression predicts outcome of interleukin 2 therapy for renal cancer. Clin Cancer Res 11: 3714-3721, 2005.

9. Bluemke K, Bilkenroth U, Meye A, et al: Detection of circulating tumor cells in peripheral blood of patients with renal cell carcinoma correlates with prognosis. Cancer Epidemiol Biomarkers Prev 18: 2190-2194, 2009.

10. McKiernan JM, Buttyan R, Bander NH, et al: The detection of renal carcinoma cells in the peripheral blood with an enhanced reverse transcriptase-polymerase chain reaction assay for $\mathrm{MN} /$ CA9. Cancer 86: 492-497, 1999.

11. de la Taille A, Katz A, Cao Y, et al: Blood-based RT-PCR assays of MN/CA9 or PSMA: clinical application in renal cancer patients. Urology 56: 393-398, 2000.
12. Gilbert SM, Whitson JM, Mansukhani M, et al: Detection of carbonic anhydrase-9 gene expression in peripheral blood cells predicts risk of disease recurrence in patients with renal cortical tumors. Urology 67: 942-945, 2006.

13. Ohlmann CH, Ozgur E, Schrader AJ, et al: Detection of circulating tumor cells in patients with renal cell carcinoma by reverse transcriptase polymerase chain reaction for G250/MNCA-9: results of a prospective trial. Urol Oncol 24: 287-293, 2006.

14. Opavsky R, Pastorekova S, Zelnik V, et al: Human MN/CA9 gene, a novel member of the carbonic anhydrase family: structure and exon to protein domain relationships. Genomics 33: 480-487, 1996.

15. Barathova M, Takacova M, Holotnakova T, et al: Alternative splicing variant of the hypoxia marker carbonic anhydrase IX expressed independently of hypoxia and tumour phenotype. Br J Cancer 98: 129-136, 2008.

16. Malentacchi F, Simi L, Nannelli C, et al: Alternative splicing variants of carbonic anhydrase IX in human non-small cell lung cancer. Lung Cancer 64: 271-276, 2009.

17. Malentacchi F, Vinci S, Melina AD, et al: Splicing variants of carbonic anhydrase IX in bladder cancer and urine sediments. Urol Oncol: Sep 25, 2010 (Epub ahead of print).

18. Zavada J, Zavadova Z, Zat'ovicova M, Hyrsl L and Kawaciuk I: Soluble form of carbonic anhydrase IX (CA IX) in the serum and urine of renal carcinoma patients. Br J Cancer 89: 1067-1071, 2003.

19. Li G, Feng G, Gentil-Perret A, Genin C and Tostain J: Serum carbonic anhydrase 9 level is associated with postoperative recurrence of conventional renal cell cancer. J Urol 180: 510-513; discussion 513-514, 2008.

20. Zhou GX, Ireland J, Rayman P,Finke J and Zhou M: Quantification of carbonic anhydrase IX expression in serum and tissue of renal cell carcinoma patients using enzyme-linked immunosorbent assay: prognostic and diagnostic potentials. Urology 75: 257-261, 2010.

21. Papworth K, Sandlund J, Grankvist K, Ljungberg B and Rasmuson T: Soluble carbonic anhydrase IX is not an independent prognostic factor in human renal cell carcinoma. Anticancer Res 30: 2953-2957, 2010.

22. Pena C, Lathia C, Shan M, Escudier B and Bukowski RM: Biomarkers predicting outcome in patients with advanced renal cell carcinoma: results from sorafenib phase III Treatment Approaches in Renal Cancer Global Evaluation Trial. Clin Cancer Res 16: 4853-4863, 2010.

23. Wind TC, Messenger MP, Thompson D, Selby PJ and Banks RE: Measuring carbonic anhydrase IX as a hypoxia biomarker: differences in concentrations in serum and plasma using a commercial enzyme-linked immunosorbent assay due to influences of metal ions. Ann Clin Biochem 48: 112-120, 2011.

24. Zatovicova M, Sedlakova O, Svastova E, et al: Ectodomain shedding of the hypoxia-induced carbonic anhydrase IX is a metalloprotease-dependent process regulated by TACE/ ADAM17. Br J Cancer 93: 1267-1276, 2005.

25. Liao SY, Aurelio ON, Jan K, Zavada J and Stanbridge EJ: Identification of the MN/CA9 protein as a reliable diagnostic biomarker of clear cell carcinoma of the kidney. Cancer Res 57: 2827-2831, 1997.

26. Sandlund J, Oosterwijk E, Grankvist K, Oosterwijk-Wakka J, Ljungberg B and Rasmuson T: Prognostic impact of carbonic anhydrase IX expression in human renal cell carcinoma. BJU Int 100: 556-560, 2007.

27. Patard JJ, Fergelot P, Karakiewicz PI, et al: Low CAIX expression and absence of VHL gene mutation are associated with tumor aggressiveness and poor survival of clear cell renal cell carcinoma. Int J Cancer 123: 395-400, 2008.

28. Wiesener MS, Munchenhagen PM, Berger I, et al: Constitutive activation of hypoxia-inducible genes related to overexpression of hypoxia-inducible factor-1alpha in clear cell renal carcinomas. Cancer Res 61: 5215-5222, 2001. 